\title{
Abstracts
}

Conclusions The IHI team learned how to work and apply the Science of Improvement in a new cultural context in Doha, Qatar. Reliably applying all elements of evidence-based care bundles can lead to improved outcomes. Healthcare delivery is complex and multiple factors effect patient outcomes. Multi-faceted approaches must be employed to reduce hospital acquired conditions. Further work should be done to develop, test and measure the effects of standard workflows for processes prone to human error and patient harm in this context.

\section{USING QUALITY IMPROVEMENT TO END HOMELESSNESS}

Beth Sandor, Jake Maguire. Community Solutions, US

10.1136/bmjoq-2017-|HI.25

Background Homelessness is an urgent public health crisis. Still, most communities do not target resources effectively to the most vulnerable homeless populations, and no framework

\section{COMMUNITY SOLUTIONS}

\section{August 2017 \\ Using Quality Improvement to End Homelessness}

Increases in Communities with Monthly By Name Data on Homeless Veterans

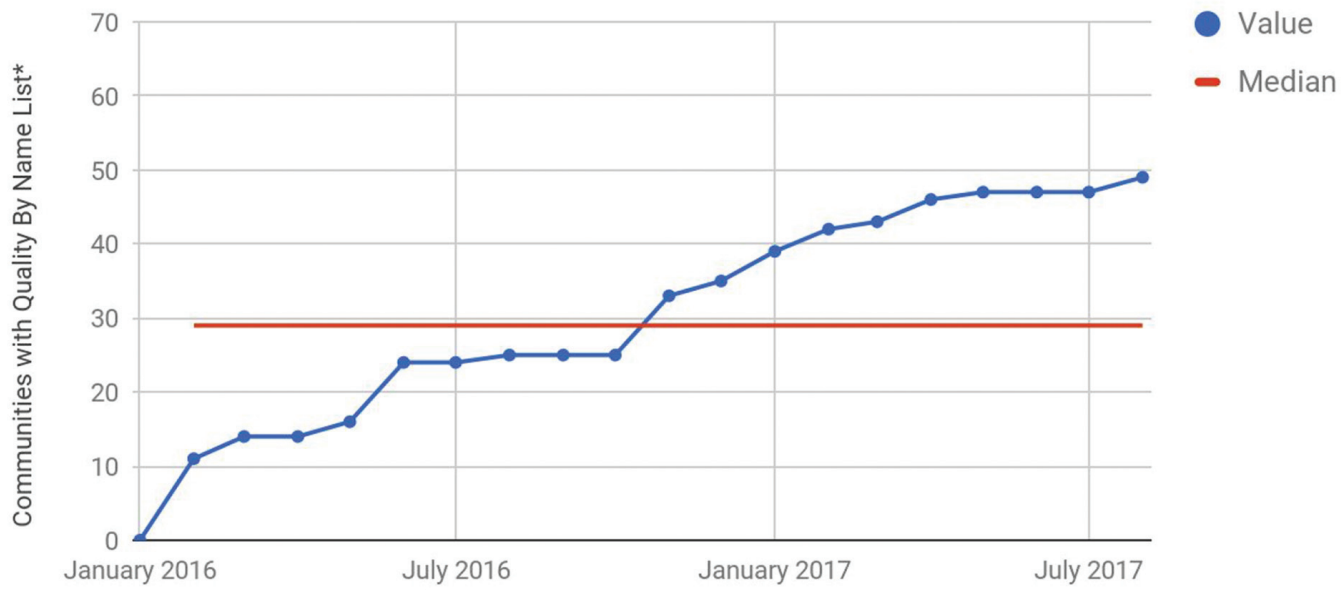

Date / Observation

*Quality By Name List is defined as a registry of all persons experiencing veteran and/or chronic homelessness across a community's entire geography, updated at least monthly.

\section{Increases in Communities with Monthly By Name Data on Chronically Homeless}

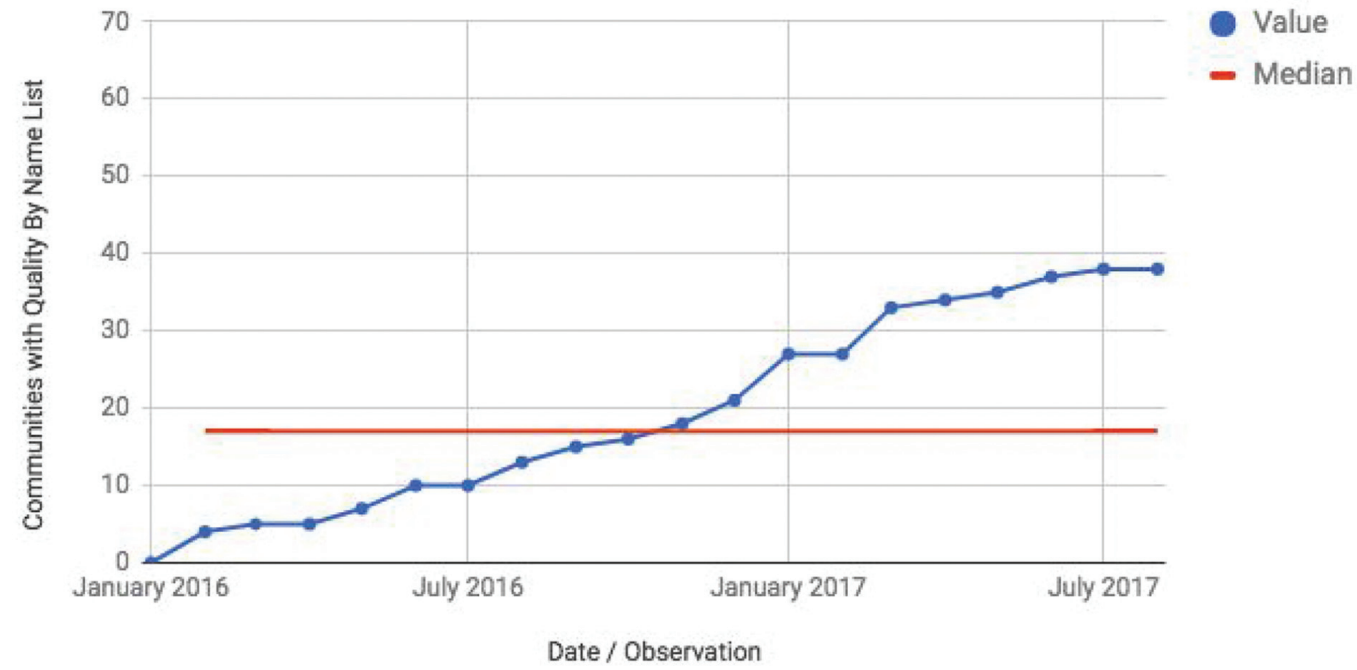




\section{COMMUNITY SOLUTIONS}

\section{Gulf Coast, MS - Ending Veteran Homelessness*}

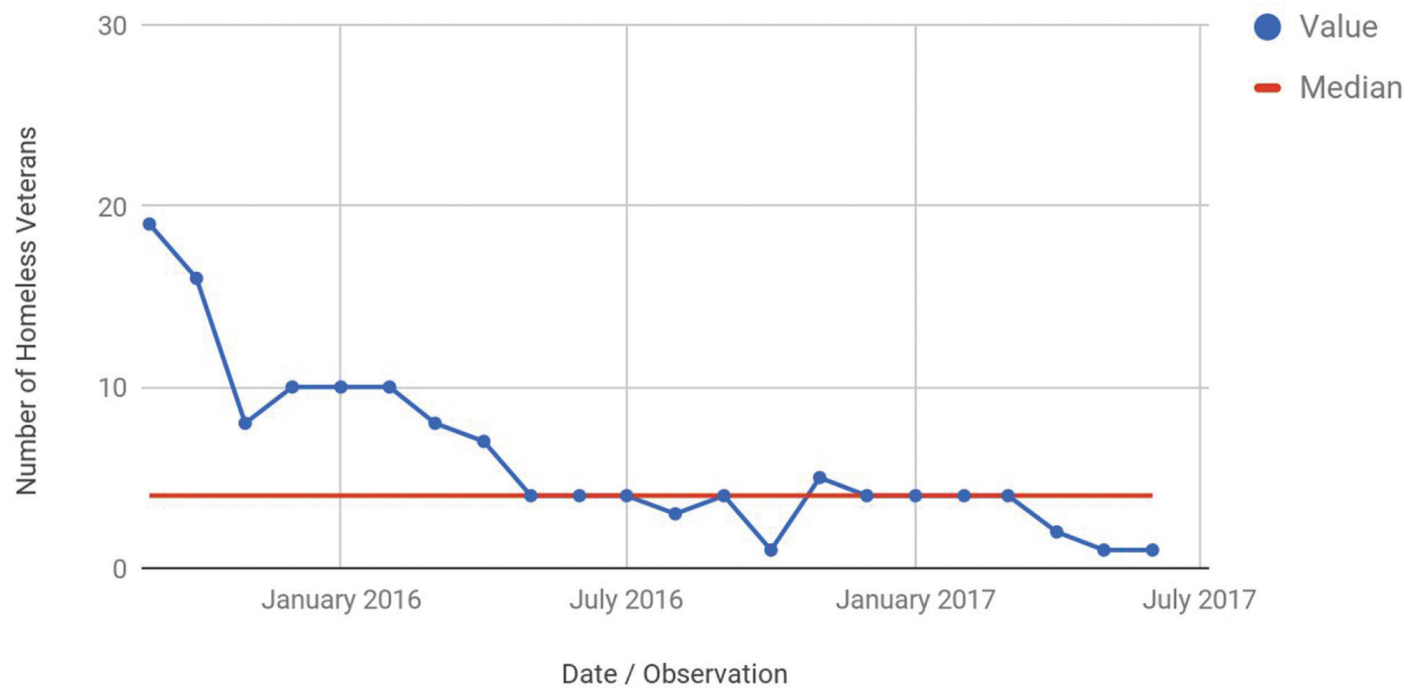

"A community has measurably ended veteran homelessness when the number of actively homeless veterans is less than that community's six-month average monthly housing placement rate.

\section{Chicago: Reductions in Number of Homeless Veterans}

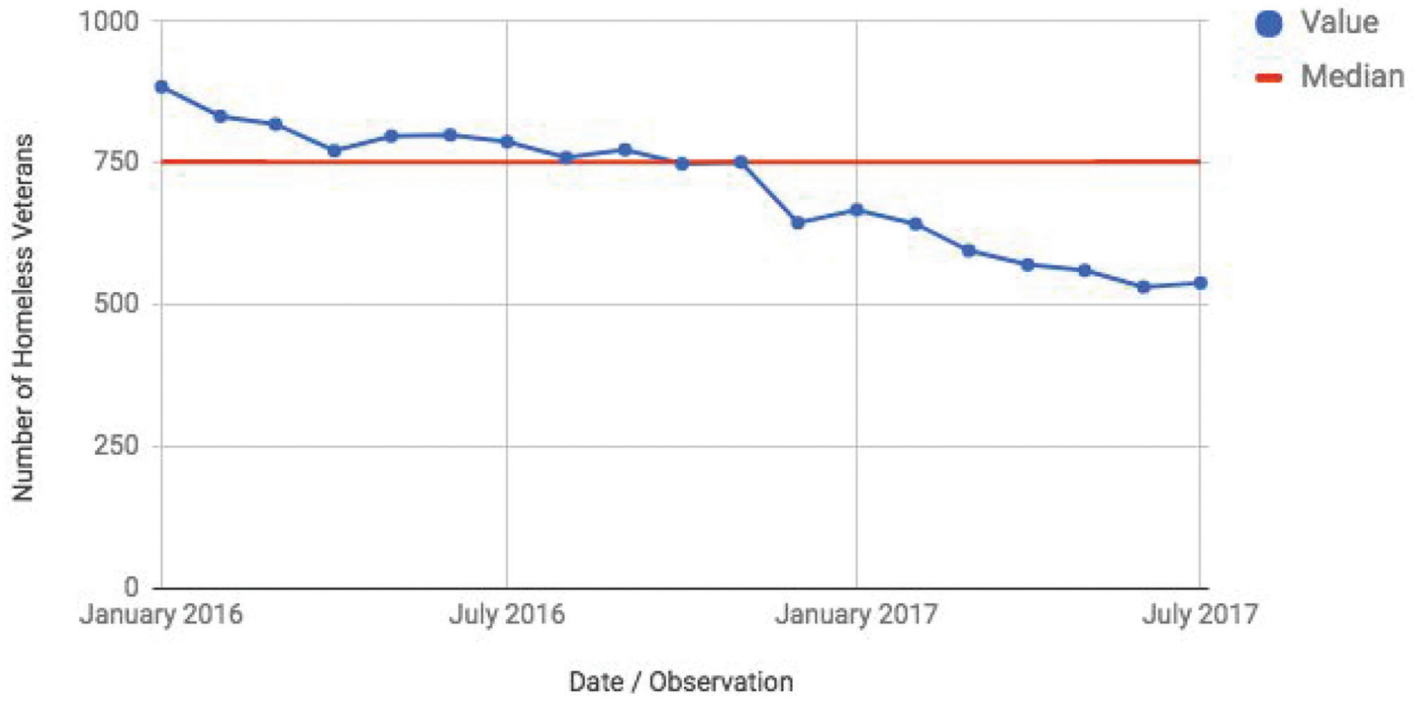

Abstract 1015 Figure 2

has traditionally existed for reducing homelessness methodically over time.

Objectives In 2010, we began working with +100 communities to increase the number of chronically homeless people being prioritised for available housing resources. In 2015, we redefined our aim to help communities end chronic and veteran homelessness outright.
Methods We have embedded quality improvement in local community teams, teaching them to set clear aims, test system interventions, and measure outcomes monthly. We initially sought to improve the number of people being housed each month. When this measure failed to correspond with reductions in homelessness, we developed a stock-and-flow benchmark for measuring an end to homelessness and asked 


\section{COMMUNITY SOLUTIONS}

\section{Bergen County, NJ - Ending Chronic Homelessness*}

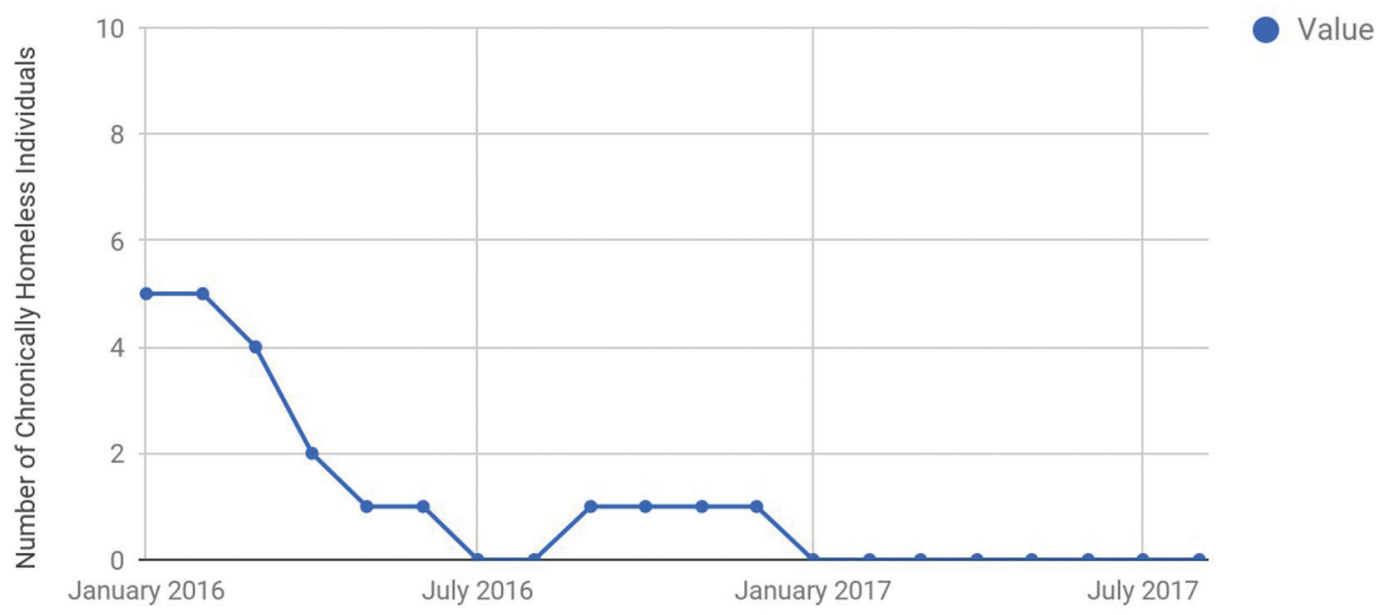

Date / Observation

"A community has measurably ended chronic homelessness when the number of individuals experiencing chronic homelessness is less than .1\% of its individual homeless population OR 3, whichever is greater.

\section{Rockford, IL - Ending Chronic Homelessness}

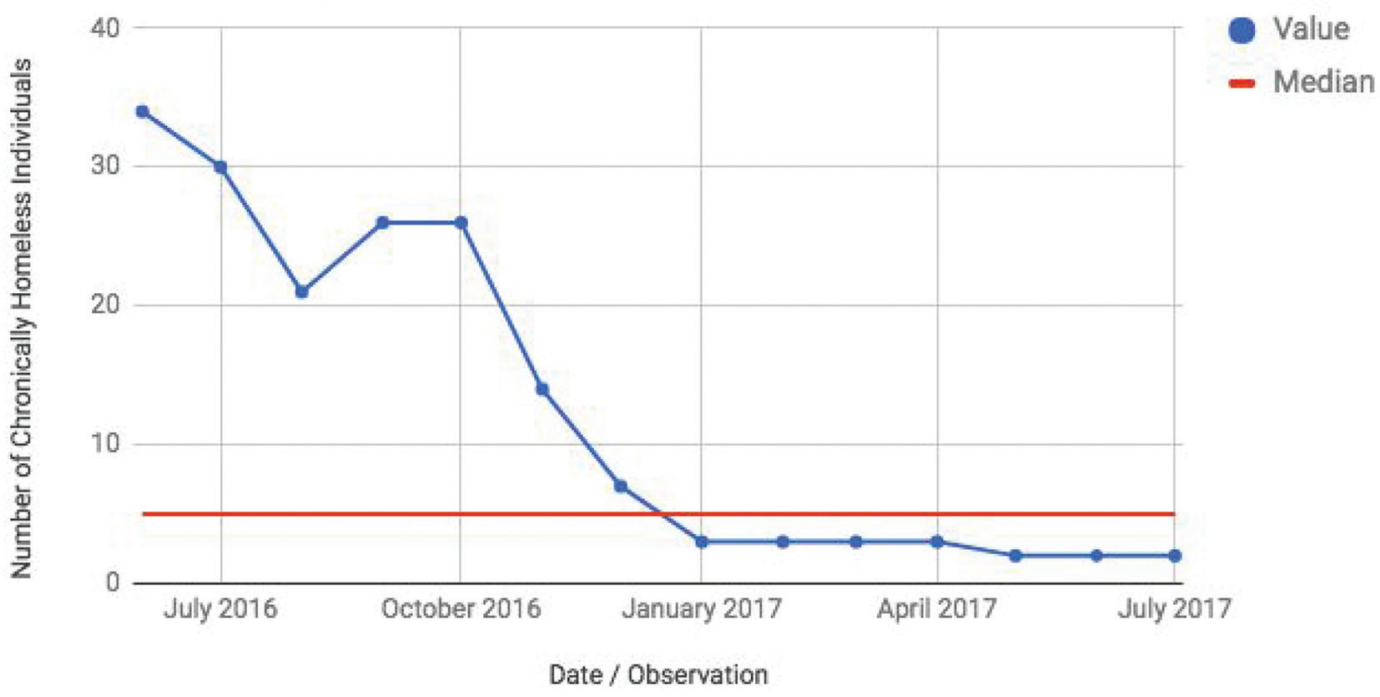

\section{Abstract 1015 Figure 3}

communities to develop and apply monthly data to measure reductions. Communities now use by-name lists of all people experiencing homelessness, updated monthly, to measure inflow into homelessness and exits to housing, and to drive reductions in homelessness overall.

Results From 2010-2014, communities increased their monthly housing rates by $266 \%$ on average, housing 105,580 people. Since we began measuring reductions, rather than housing rates, 55 communities have achieved monthly data on homelessness, 30 are reducing homelessness month-over-month, and ten have ended chronic and/or veteran homelessness.

Conclusions Reducing homelessness requires a multi-variate improvement framework, inclusive of inflow, housing rates, and current rates of homelessness. By tracking and responding 
to these variables monthly, communities can diagnose problems more precisely and identify improvements more quickly.

\section{EVALUATION OF THE EDMONTON ZONE TRIPLE AIM INITIATIVE: BUILDING AND IMPLEMENTING A MEASUREMENT SYSTEM FOR IMPROVEMENT WITH COMPLEX, VULNERABLE CLIENTS}

Eric VanSpronsen, Christine Vandenberghe, Melanie Hennig, Tristan Robinson, Lana Socha, SungHyun Kang, Xiaoming Wang, Lorraine Telford, Dorah Conteh. Alberta Health Services, Canada

\subsection{6/bmjoq-2017-|HI.26}

Background The Alberta Health Services, Edmonton Zone Triple Aim Initiative launched in January, 2013. The seven participating clinical teams target complex, vulnerable patients in inner-city Edmonton, Alberta, Canada. The Initiative follows the Institute for Healthcare Improvement's Better Health Lower Cost Road Map and pursues the Quadruple Aim. Grant funding was provided by MERCK Canada and Alberta Innovates.

Objectives The objectives of the evaluation were: 1) Determine the extent to which the Aims of the Initiative have been met; and 2) Build a measurement system to monitor the performance of the team quality improvement efforts.

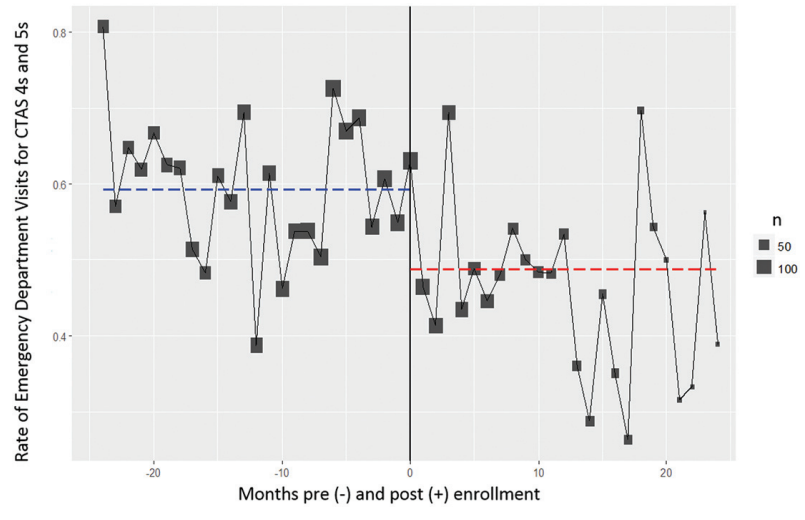

CTAS= Canadian Triage \& Acuity Scale (CTAS $4 \& 5$ are less acute)

Abstract 1033 Figure 1 Average monthly rates for emergency department visits for CTAS $4 \mathrm{~s}$ and $5 \mathrm{~s}$. CTAS=Canadian Triage and Acuity Scale (CTAS 4 and 5 are less acute)

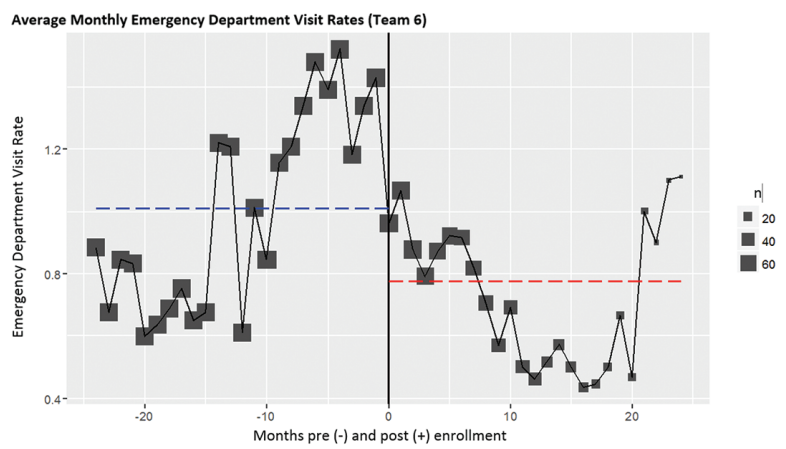

Abstract 1033 Figure 2 Average monthly emergency department visit rates (team 6)

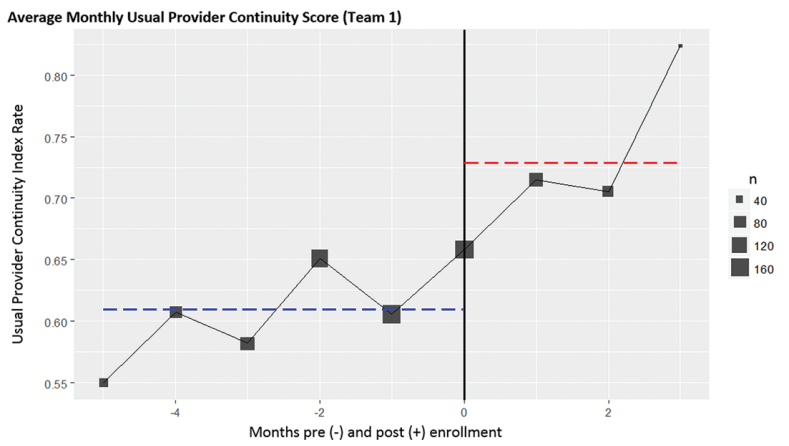

Abstract 1033 Figure 4 Average monthly usual provider continuity score (team 1)

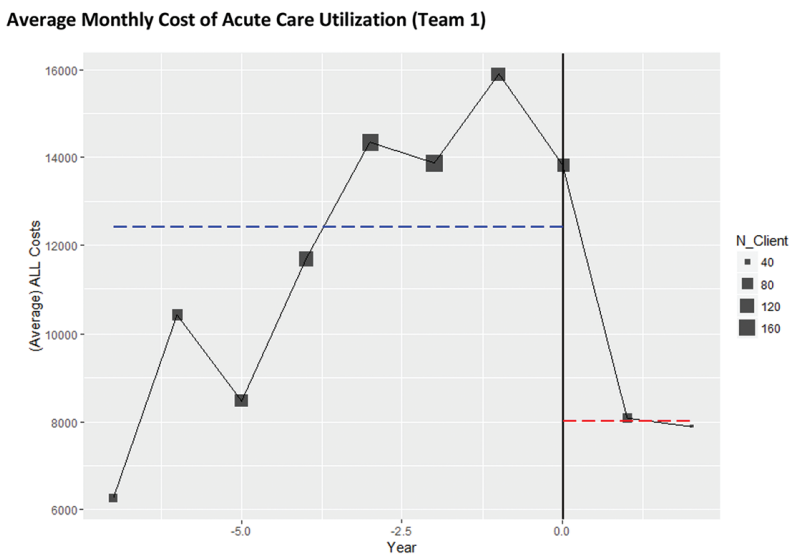

Abstract 1033 Figure 5 Average monthly cost of acute care utilisation (team 1)

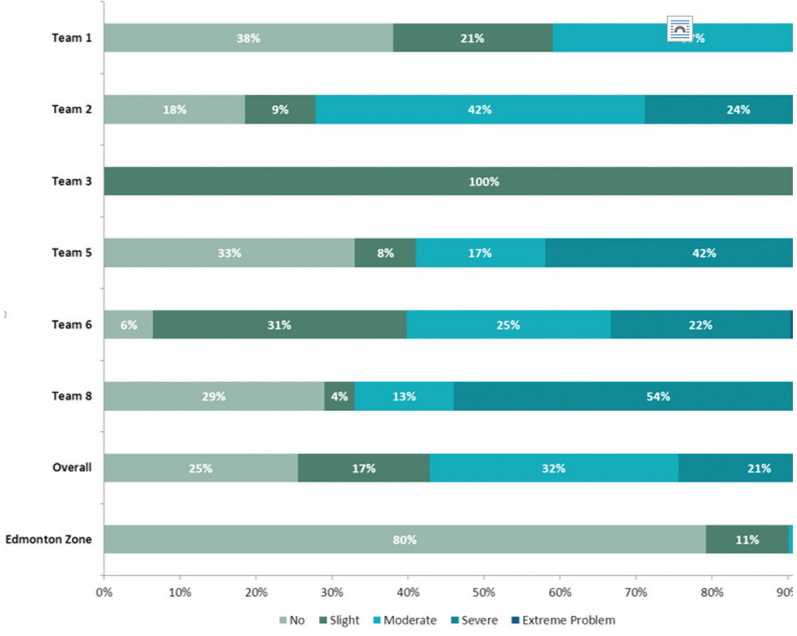

Abstract 1033 Figure 6 EQ-5D-5L Mobility dimension responses

Methods Over 40 providers and 445 patients participated. Data collection include: administrative data; patient surveys; and patient and staff interviews. Analyses of system level data (e.g., emergency department visits; inpatient stays; and 